\title{
From triple-point materials to multiband nodal links
}

\author{
Patrick M. Lenggenhager $\odot,{ }^{1,2,3, *}$ Xiaoxiong Liu $\odot,{ }^{3}$ Stepan S. Tsirkin $\odot,{ }^{3}$ Titus Neupert $\odot,{ }^{3}$ and Tomáš Bzdušek $\odot{ }^{1,3}$ \\ ${ }^{1}$ Condensed Matter Theory Group, Paul Scherrer Institute, 5232 Villigen PSI, Switzerland \\ ${ }^{2}$ Institute for Theoretical Physics, ETH Zurich, 8093 Zurich, Switzerland \\ ${ }^{3}$ Department of Physics, University of Zurich, Winterthurerstrasse 190, 8057 Zurich, Switzerland
}

(Received 24 August 2020; revised 5 January 2021; accepted 10 February 2021; published 1 March 2021)

\begin{abstract}
We study a class of topological materials which in their momentum-space band structure exhibit threefold degeneracies known as triple points. Focusing specifically on $\mathcal{P} \mathcal{T}$-symmetric crystalline solids with negligible spin-orbit coupling, we find that such triple points can be stabilized by little groups containing a three-, four-, or sixfold rotation axis, and we develop a classification of all possible triple points as type A vs type B according to the absence vs presence of attached nodal-line arcs. Furthermore, by employing the recently discovered non-Abelian band topology, we argue that a rotation-symmetry-breaking strain transforms type-A triple points into multiband nodal links. Although multiband nodal-line compositions were previously theoretically conceived and related to topological monopole charges, a practical condensed-matter platform for their manipulation and inspection has hitherto been missing. By reviewing the known triple-point materials with weak spin-orbit coupling and by performing first-principles calculations to predict new ones, we identify suitable candidates for the realization of multiband nodal links in applied strain. In particular, we report that an ideal compound to study this phenomenon is $\mathrm{Li}_{2} \mathrm{NaN}$, in which the conversion of triple points to multiband nodal links facilitates a largely tunable density of states and optical conductivity with doping and strain, respectively.
\end{abstract}

DOI: 10.1103/PhysRevB.103.L121101

Introduction. The recent rapid developments in the topological band theory of crystals [1-12] and engineered metamaterials [13-17] fueled a fruitful research into topological band degeneracies in semimetals and metals. Currently, nodal lines (NLs) [18-21] exhibiting a quantized Zak-Berry phase $[22,23]$ are arguably the most investigated type of band degeneracy and were found to form intricate structures, including chains, links, and knots [24-27]. More recently, threefold-degenerate nodal points [28-33], also called triple points (TPs), received attention as peculiar intermediates between Weyl and Dirac points [34-42]. For spin-orbit-coupled (SOC) systems, TPs were classified into type A vs type B according to the absence or presence of attached nodal-line arcs [28], but they were also reported to occur in certain materials with negligible SOC [43-47], including Bernal graphite [48].

Curiously, the topological stability of both NLs and TPs is fully captured only if one partitions the energy bands via multiple energy gaps. On the one hand, NLs in spacetime-inversion $(\mathcal{P T})$ symmetric systems with negligible SOC (i.e., "spinless") exhibit Euler and Stiefel-Whitney monopole charges [49-52], which are related to linking with NLs inside adjacent energy gaps $[53,54]$. On the other hand, TPs formed by a crossing of a one-dimensional (1D) and a twodimensional (2D) irreducible corepresentation (ICR) along a rotation axis are end points at which a NL is transferred between two adjacent energy gaps. Multiband nodal structures in $\mathcal{P T}$-symmetric spinless systems were recently characterized by non-Abelian quaternion invariants [55-59], which

*Corresponding author: lenpatri@ethz.ch generalize the Zak-Berry-phase quantization. However, practical condensed-matter platforms to manipulate and probe the non-Abelian band topology have remained limited.

Here we investigate the interplay of TPs and non-Abelian band topology, and we suggest a platform to experimentally observe multiband nodal links in TP materials under strain. To obtain these results, we develop a symmetry-based classification of TPs in spinless $\mathcal{P T}$-symmetric systems into type A or B (Fig. 1) similar to the SOC case. By employing the novel insights of non-Abelian quaternion charges, we then illuminate a relation between the NL and TP topology; in particular, we show that type-A TPs in spinless $\mathcal{P T}$-symmetric models generally evolve into multiband nodal links (Fig. 2) under rotation-symmetry-breaking perturbations [60]. After reviewing the known spinless TP materials and by performing first-principles calculations to identify additional ones, we finally predict that the recently reported triple-point metal $\mathrm{Li}_{2} \mathrm{NaN}$ [46] produces an ideal multiband nodal link in applied strain, with the node conversion manifested by unusual strain-tunable optical conductivity. Our results suggest a concrete material platform for experimental studies of nonAbelian band topology and pave the way to uncover further links between seemingly unrelated topological band features.

Triple points and non-Abelian band topology. TPs are symmetry-protected crossings of a $1 \mathrm{D}$ ICR and a $2 \mathrm{D}$ ICR occurring along rotation-invariant lines in $\mathbf{k}$ space [Fig. 2(a)] [28]. It is possible to recast the corresponding 2D ICR as a NL, in which case the TP marks a location where the NL is transferred from one energy gap (i.e., one pair of energy bands) to an adjacent one [Fig. 2(c)]. In this work we consider $\mathcal{P} \mathcal{T}$-symmetric systems with negligible SOC (i.e., 


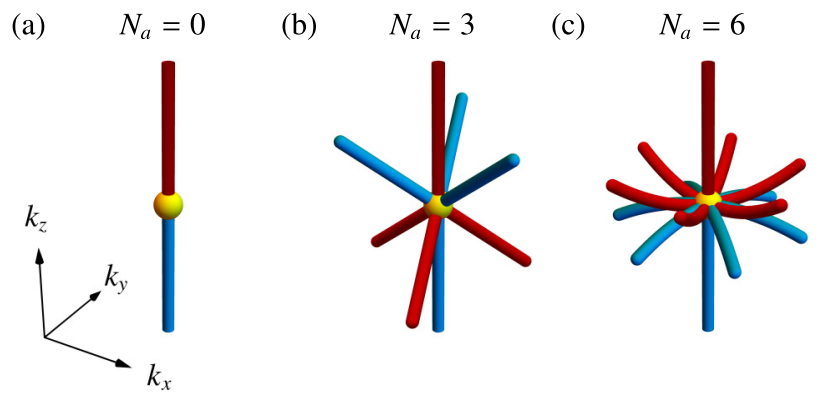

FIG. 1. Three species of triple points in spinless $\mathcal{P} \mathcal{T}$-symmetric systems. They are characterized by the number $N_{a}$ of attached NL arcs, which depends on symmetry as listed in Table I. We adopt terminology of Ref. [28], and call TPs with $N_{a}=0(\neq 0)$ type A (type B).

spinless), such that $(\mathcal{P T})^{2}=+\mathbb{1}$ [49]. A mathematical theorem [57] then implies a basis in which the Bloch states are real (rather than complex) vectors. Under these circumstances, topological properties of multiband nodal-line compositions are captured within the framework of non-Abelian band topology [55].

While the Zak-Berry phase $\varphi_{\mathrm{ZB}}$ (quantized by $\mathcal{P} \mathcal{T}$ to zero vs $\pi$ on closed paths) of a block of bands remains invariant provided that these bands are separated by an energy gap from other groups of bands, the non-Abelian invariant (called the generalized quaternion charge by Ref. [55]) $\mathfrak{q} \in \mathrm{Q}_{N}$ of an $N$-band system is susceptible to closing any of the $(N-1)$ energy gaps [labeled 1 to $(N-1)$ according to increasing energy] between consecutive bands. If $\varphi_{\mathrm{ZB}}^{j}(\gamma)=\pi$ for some band $j$ on path $\gamma$, then the square $\mathfrak{q}^{2}(\gamma)=-1 \in Q_{N}$ is the unique nontrivial element that squares to identity $+1 \in \mathrm{Q}_{N}$ [54]. For $\mathfrak{q}\left(\gamma^{\prime}\right)=-1$ all energy bands on $\gamma^{\prime}$ have a trivial Zak-Berry phase; nevertheless, there is a topological obstruction corresponding to an overall $2 \pi$ rotation of the $N$ frame (the eigenframe) spanned by the Bloch states [57].
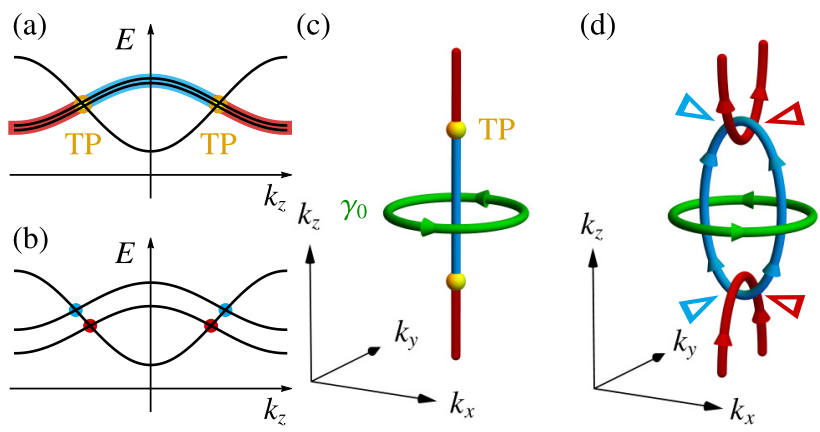

FIG. 2. Relation of type-A triple points (TPs) to multiband nodal links. Nodal lines (NLs) in the first (second) band gap are displayed in red (blue), and TPs are in yellow. (a) and (b) Band structure along a high-symmetry line and (c) and (d) the NL composition near the TPs before and after applying rotation-symmetry-breaking perturbation, respectively. The green path $\gamma_{0}$ carries quaternion charge -1 . The orientations of the NLs (with reversals indicated by triangles of the corresponding color) follow the convention of Ref. [54].
Multi-band nodal links. We argue that if type-A TPs were to arise in a spinless $\mathcal{P} \mathcal{T}$-symmetric system, they must necessarily evolve into multiband nodal links under rotationsymmetry-breaking strain. To understand this phenomenon, consider a loop $\gamma_{0}$ [green in Fig. 2(c)] that encircles the NL [blue in Fig. 2] extending to one side of a TP (yellow in Fig. 2). To study the stability of the NL, one could compute the Zak-Berry phase $\varphi_{\mathrm{ZB}}\left(\gamma_{0}\right)$ [Fig. 2(c)] assuming a gap between the two bands that meet at the NL. For a type-A TP it must hold that $\varphi_{\mathrm{ZB}}\left(\gamma_{0}\right)=0$ because the loop can be slid to the other side of the TP, where it can be trivialized without closing the corresponding energy gap. However, from the perspective of the non-Abelian invariant the path $\gamma_{0}$ cannot be shrunk to a point on the other side of the TP due to the presence of the NL (red in Fig. 2) in the adjacent energy gap. This suggests a nontrivial value $\mathfrak{q}\left(\gamma_{0}\right) \neq+1$ characterizing the NL composition around the type-A TP.

The outlined characterization of the NLs is consistent with the 2D ICR being a double nodal line, i.e., one with a quadratic splitting of the two energy bands away from the high-symmetry line [45]. In that case, the Zak-Berry phase on $\gamma_{0}$ would be trivial, but the quaternion charge would exhibit the nontrivial value $\mathfrak{q}\left(\gamma_{0}\right)=-1$ [55]. Crucially, the non-Abelian invariant remains well defined even after the 2D ICR is split by a rotation-symmetry-breaking perturbation [Fig. 2(b)], when the TP is lost. While the $\mathbb{Z}_{2}$-valued Zak-Berry phase $\varphi_{\mathrm{ZB}}\left(\gamma_{0}\right)=0$ cannot be directly applied to predict the fate of the NL composition upon such a symmetry breaking, the value $\mathfrak{q}\left(\gamma_{0}\right)=-1$ implies that $\gamma_{0}$ must enclose a pair of NLs in some energy gap. It therefore follows that the symmetry breaking transforms the TP into a multiband nodal link extending around the Brillouin zone [Fig. 2(d)]. The topological stability of the pair of NLs implies that they carry parallel orientation, which is consistent with the noncommutativity of the non-Abelian invariant [54].

Classification of TPs in spinless band structures. The discussed relation between TPs and multiband nodal links relies on (i) the 2D ICR carrying a nontrivial quaternion charge, $\mathfrak{q}=-1$, and (ii) the TP being type A. We therefore develop a classification of symmetry-protected TPs in spinless $\mathcal{P} \mathcal{T}$ symmetric systems according to those two aspects. We restrict our analysis to nonmagnetic space groups (SGs) and to highsymmetry lines whose ICRs are not affected by a possible nonsymmorphicity of the SG.

To obtain the classification, we consider a rotationinvariant line in $\mathbf{k}$ space (without loss of generality along $k_{z}$ ) of some SG and denote its little group by $\mathcal{G}$; then, under the above restrictions, $\mathcal{G}$ is a nonmagnetic point group with a single rotation axis and without inversion. By assumption the system is $\mathcal{P T}$ symmetric, such that the relevant symmetry group is enhanced to the nonunitary $\mathcal{M}=\mathcal{G} \cup(\mathcal{P T}) \mathcal{G}$. The stability of a TP requires $\mathcal{M}$ to support both 1D and 2D ICRs, which is the case for $\mathcal{G} \in\left\{C_{3(v)}, C_{4(v)}, C_{6(v)}\right\}$ [61]. To classify the possible TPs we construct minimal two-band (three-band) $\mathbf{k} \cdot \mathbf{p}$ models for all 2D ICRs (combinations of 1D + 2D ICRs) of each $\mathcal{G}$ mentioned above. For that we obtain the necessary IRs from the Bilbao crystallographic server [62-64], then construct the corresponding ICRs [61] and finally obtain the models using the PYTHON package KDOTP-SYMMETRY [65]. 
TABLE I. Result of the classification of triple points (TPs) in spinless $\mathcal{P} \mathcal{T}$-symmetric models (assumed to be nonmagnetic and symmorphic). The TP species [characterized by the winding number $w_{2 \mathrm{D}}$ of the 2D irreducible corepresentation (ICR) and the number $N_{a}$ of attached NL arcs per gap] depends on the little group $\mathcal{G}$. The TP is type A if $N_{a}=0$ (and type B otherwise). For $C_{6(v)}$ the result further depends on the pair of intersecting ICRs, where $i \in\{1,2\}$ for $C_{6 v}$. The notation of the ICRs follows Ref. [61]. TPs with $\left|w_{2 \mathrm{D}}\right|=2$ carry quaternion charge $\mathfrak{q}=-1$, and when $N_{a}=0$, they transform to multiband nodal links under strain. The last column reviews previously reported light-element TP materials.

\begin{tabular}{|c|c|c|c|c|c|}
\hline $\mathcal{G}$ & Pairs of ICRs | & $\left|w_{2 \mathrm{D}}\right|$ & $N_{a}$ & type & Material candidates \\
\hline$C_{3}$ & $($ any $2 \mathrm{D}+1 \mathrm{D})$ & 1 & 3 & $\mathrm{~B}$ & $\mathrm{Na}_{2} \mathrm{O}^{\mathrm{a}}$ \\
\hline$C_{3 v}$ & $($ any $2 \mathrm{D}+1 \mathrm{D})$ & 1 & 3 & B & Bernal graphite [48] \\
\hline$C_{4}$ & $($ any $2 \mathrm{D}+1 \mathrm{D})$ & 2 & 0 & A & \\
\hline$C_{4 v}$ & (any $2 \mathrm{D}+1 \mathrm{D})$ & 2 & 0 & A & $\mathrm{ZrO}[44], \mathrm{Sc}_{3} \mathrm{GaC}[45], \mathrm{CaNaP}^{2}$ \\
\hline$C_{6}$ & $\left(E_{2}, A\right),\left(E_{1}, B\right)$ & 2 & 0 & A & \\
\hline$C_{6}$ & $\left(E_{1}, A\right),\left(E_{2}, B\right)$ & 2 & 6 & $\mathrm{~B}$ & \\
\hline$C_{6 v}$ & $\left(E_{1}, A_{i}\right),\left(E_{2}, B_{i}\right)$ & 2 & 0 & A & $\mathrm{Li}_{2} \mathrm{NaN}$ [46], $\mathrm{TiB}_{2}[43]$ \\
\hline$C_{6 v}$ & $\left(E_{2}, A_{i}\right),\left(E_{1}, B_{i}\right)$ & 2 & 6 & $\mathrm{~B}$ & $\mathrm{Na}_{3} \mathrm{~N}[47], \mathrm{AlN}^{\mathrm{a}}$ \\
\hline
\end{tabular}

${ }^{\mathrm{a}}$ Compound reported and inspected in Ref. [64].

The constructed models are available in the supplementary data [66].

For two-band spinless $\mathcal{P} \mathcal{T}$-symmetric models the Hamiltonian is of the form $\mathcal{H}(\mathbf{k})=h_{x}(\mathbf{k}) \sigma_{x}+h_{z}(\mathbf{k}) \sigma_{z}$ [49]; therefore, we can define the winding number $w_{2 \mathrm{D}} \in \mathbb{Z}$ of the vector $\mathbf{h}=\left(h_{x}, h_{z}\right)$ [18] for a closed loop $\gamma_{0}$ [Fig. 2(c)] around the NL corresponding to the 2D ICR. While the winding number is unstable upon the addition of trivial bands, partial topological information is preserved in the quaternion charge. In particular, there is a reduction $w_{2 \mathrm{D}}=2 \bmod 4 \Rightarrow \mathfrak{q}\left(\gamma_{0}\right)=$ -1 corresponding to the stability of a $2 \pi$ rotation of the eigenframe [57]. The winding number can be analytically calculated for all the constructed two-band $\mathbf{k} \cdot \mathbf{p}$ models.

Finally, using methods detailed in a future work by us [68], we characterize the TPs according to the number $N_{a}$ of additional attached NLs (which we call NLarcs) per band gap. Following the terminology of Ref. [28] for the SOC case, we call TPs with $N_{a}=0(\neq 0)$ type A (type B). In particular, we analyze the NL compositions of the three-band models, which are fully determined by real roots of the discriminant of the characteristic polynomial of the Hamiltonian [67]. For a given group $\mathcal{G}$ the possible combinations of ICRs fall into at most two topologically distinct equivalence classes.

The result of our analysis (Table I) includes TPs with $N_{a} \in\{0,3,6\}$ with attached arcs universally scaling as $k_{z}=$ $\left(k_{x}^{2}+k_{y}^{2}\right)^{\left|w_{2 D}\right| / 2}$ near the TP (Fig. 1). While the precise NL composition depends on the parameters of the $\mathbf{k} \cdot \mathbf{p}$ model, we find the characteristics $\left|w_{2 \mathrm{D}}\right|$ and $N_{a}$ (type) of a given TP class to be universal. In particular, we find that $\mathcal{G} \in\left\{C_{4(v)}, C_{6(v)}\right\}$ enables type-A TPs with $\mathfrak{q}=-1$, which evolve into multiband nodal links when subjected to rotation-symmetry-breaking strain. Table I further lists previously reported TPs in spinless $\mathcal{P} \mathcal{T}$-symmetric compounds [43-48], as well as three newly predicted materials which we identified using first-principles calculations. We analyze those compounds in Ref. [68] and verify the agreement with our predictions.

Multiband nodal link in strained $\mathrm{Li}_{2} \mathrm{NaN}$. Hexagonal $\mathrm{Li}_{2} \mathrm{NaN}$ (entry \#92308 in the Inorganic Crystal Structure Database; see Table I) was reported to be a "superior" TP material with negligible SOC [46]. In particular, it exhibits a pair of type-A TPs at the Fermi level which we find to carry $\mathfrak{q}=-1$ and no other coexisting Fermi surfaces. The material has a centrosymmetric hexagonal crystal structure with SG P6/mmm (No. 191) [Fig. 3(a)]. Additionally, the corresponding 2D ICR has a nearly flat dispersion along the rotation axis [Fig. 3(b), path $\Gamma A]$, which results in a nearly hundredfold change in the density of states within a narrow energy range around the Fermi level (Fig. 3(j); see the Supplemental Material (SM) [69]). We therefore propose $\mathrm{Li}_{2} \mathrm{NaN}$ as an ideal candidate to study the conversion of the TP into a multiband nodal link, and we argue this conversion is manifested in sensitive strain-tunable optical conductivity as discussed below.

We employ first-principles calculations to study $\mathrm{Li}_{2} \mathrm{NaN}$. The bands close to the Fermi level come mainly from nitrogen $p_{x, y, z}$ and sodium $s$ orbitals; thus, we construct a corresponding four-band tight-binding model that reproduces the low-energy theory of $\mathrm{Li}_{2} \mathrm{NaN}$ [Fig. 3(b)] after being fitted to first-principles data (see the SM [69]). We find that the vertical NL at $k_{x}=0=k_{y}$ (i.e., the $2 \mathrm{D}$ ICR) is well isolated from other NLs in the Brillouin zone (BZ), both in energy and in momentum [Figs. 3(b) and 3(c)]. The little group along $\Gamma A$ is $C_{6 v}$, and the involved ICRs are $\left(E_{1}, A_{1}\right)$, such that Table I predicts $N_{a}=0$ and the formation of a multiband nodal link after breaking the $C_{6 v}$ symmetry down to $C_{2 v}$. In the tight-binding description we model this by adding an energy offset $\delta E= \pm s$ to nitrogen $p_{x, y}$ orbitals. In agreement with Figs. 2(c) and 2(d), we find that the central NL splits into linked NLs in adjacent band gaps [Figs. 3(c)-3(f)] with width in the $k_{x / y}$ direction proportional to $\sqrt{s}$.

We next examine the bulk-boundary correspondence, which has not yet been clarified for the quaternion charges. We consider a finite slab with the surface termination indicated in Fig. 3(a). This corresponds to a projection of the BZ along the reciprocal vector $\mathbf{G}_{1}$ [Fig. 3(c)] onto the surface Brillouin zone (SBZ). Each point in the SBZ corresponds to a closed path in the BZ, carrying quaternion charge $\mathfrak{q}\left(k_{2}, k_{3}\right)$ [Fig. 3(g)]. Recall [54] that any element of $Q_{N}$ is given (up to the sign) by an ordered product of some of the $(N-1)$ generators $g_{j}$, where $g_{j}$ represents band inversion of the energy gap $j$ [see legend to Fig. 3(g)]. We observe in the surface spectrum along $k_{2}=0$ two clear surface bands [Fig. 3(h)]. Additional surface bands that hybridize with the bulk states in the second gap are not discernible for small strain. A careful analysis (see the SM [69]) reveals that the surface states appear exactly in the gaps with a band inversion according to the value of $\mathfrak{q}$ when we include those that have hybridized with bulk states.

The NLs at the Fermi level and their splitting into multiband nodal links suggest nontrivial transport signatures in $\mathrm{Li}_{2} \mathrm{NaN}$. Using the Kubo-Greenwood formula $[70,71]$ which we have implemented in the PYTHON package WANNIERBERRI [72] as will be described elsewhere, we compute the interband (i.e., optical) conductivity $\sigma_{\alpha \beta}(\omega)$ [Fig. 3(i)] with and without strain for the fitted tight-binding model. We observe that 

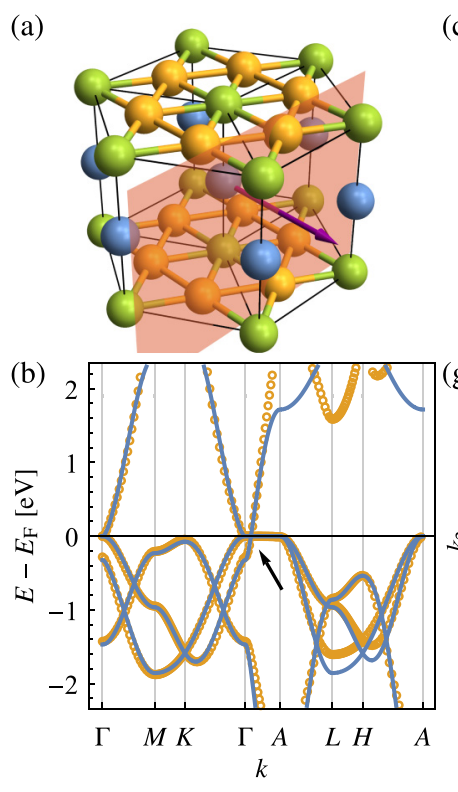

(c)
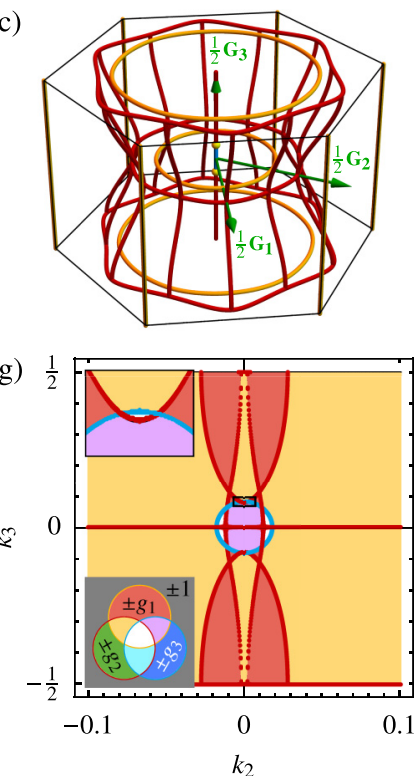

(d)

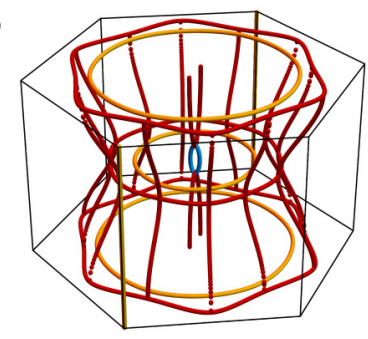

(m) (e)

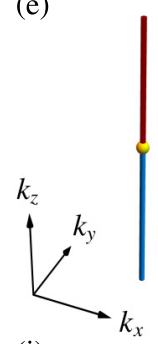

(f)

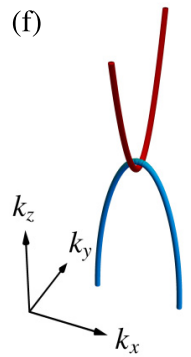

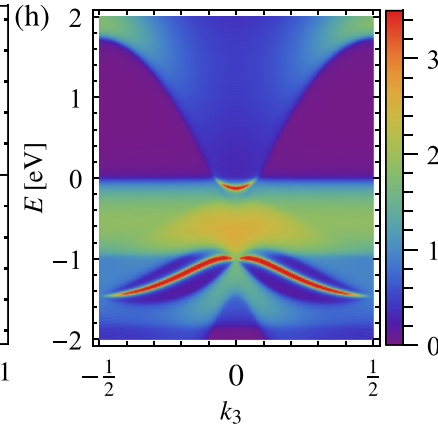

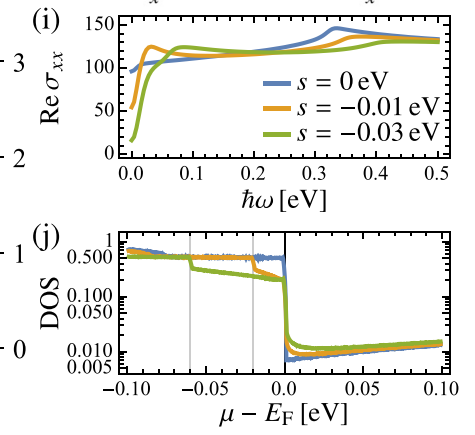

FIG. 3. $\mathrm{Li}_{2} \mathrm{NaN}$. (a) Crystal structure with $\mathrm{Na}$ (blue), Li (yellow), and $\mathrm{N}$ (green) atoms. The red plane indicates the surface termination of (g) and (h), and the purple arrow is its normal. (b) Band structure along high-symmetry lines: first-principles data (orange points) and fitted four-band tight-binding model (blue lines). The black arrow marks the triple point. (c) and (d) NLs inside the Brillouin zone without and with strain, respectively, and (e,f) close-ups for $0<k_{z}<0.15 G_{3}$, colored orange, red, and blue for the first, second, and third energy gaps, respectively. Green arrows in (c) labeled $\mathbf{G}_{1,2,3}$ indicate primitive reciprocal vectors. (g) Projection of the NLs in (d) onto the surface Brillouin zone and the quaternion charge in each region (see the text). Coordinates $k_{2,3}$ and their units correspond to projections of $\mathbf{G}_{2,3}$. (h) Surface spectral function for $k_{2}=0$. (i) and (j) Interband optical conductivity $\operatorname{Re} \sigma_{x x}$ (in $\mathrm{S} / \mathrm{cm}$ ) and density of states (DOS; in $\mathrm{eV}^{-1}$ ) computed from the fitted tight-binding model for the indicated values of strain $s$ [69]. In (d) and (f)-(h) we set $s=-0.01 \mathrm{eV}$, which corresponds to tensile strain in the $y$ direction of $\approx 1 \%$.

application of strain leads to strong suppression of $\Re \sigma_{x x}$ for infrared frequencies $\hbar \omega \lesssim 2|s|$. This results from a suppression of optical transitions due to strain-induced mismatch between the chemical potential and the energy of the NLs [73] because at fixed filling the applied strain moves the blue (red) NL in Figs. 3(e) and 3(f) above (below) the chemical potential. The strain dependence of other components of the optical conductivity tensor is presented in the SM [69].

Besides the interband contributions to conductivity, in practice one may need to also consider the intraband (Boltzmann) conductivity. While the material's relaxation time which determines this contribution is inherently difficult to predict from first principles, the intraband conductivity is dependent on the density of states at the Fermi level. Since the latter quantity does not exhibit a noticeable growth in applied strain [see Fig. 3(j)], we expect the strain-induced suppression of low-frequency conductivity to persist in such a more complete analysis.

Conclusions and outlook. We enriched the library of cataloged band-structure nodes by classifying TPs in spinless systems as type A or B and by predicting via non-Abelian band topology the conversion of type-A TPs into multiband nodal links in symmetry-breaking strain. We argued that for "ideal" TP materials (i.e., with nondispersive 2D ICR at the Fermi level along the rotation axis, such as predicted in $\mathrm{Li}_{2} \mathrm{NaN}$ ), this conversion facilitates a rapidly strain tunable optical conductivity. We anticipate the non-Abelian band topology to be analogously applicable to study conversions of other species of band nodes, including nodal chains and type-B TPs. This strategy was very recently exemplified by the predicted Weyl-points-to-nodal-ring conversion in $\mathrm{ZrTe}$ $[57,74]$. We emphasize the generality of this framework, as the "spinlessness" condition $(\mathcal{P T})^{2}=+\mathbb{1}$ extends to photonic, phononic, and magnonic bands.

We expect the nontrivial linking to furnish the NL rings discussed here with monopole charges [49-54]. In particular, braiding rules of Ref. [51] suggest that the red NL of $\mathrm{Li}_{2} \mathrm{NaN}$ in Figs. 3(e) and 3(f) should carry Euler class $\chi=2$. Note that nodal rings with monopole charges were theoretically related to higher-order topology with hinge Fermi arcs [75-77], yet suitable material candidates at present remain very scarce [52]. Our findings suggest that hinge Fermi arcs may arise in spinless triple-point materials; therefore, identifying general conditions for the realization of nontrivial Euler or Stiefel-Whitney monopole charges in TP-materials suggests an interesting future application of our results. Data and code are available from ETH Zurich [66].

Acknowledgments. We thank A. A. Soluyanov for valuable discussions and Q. S. Wu for providing early data that facilitated our work on this project. We further acknowledge discussions and helpful comments on the manuscript from F. O. von Rohr, R.-J. Slager, L. Trifunovic, and N. A. Winter. P.M.L. and T.B. were supported by the Ambizione Grant No. 185806 from the Swiss National Science Foundation. T.N. and S.S.T. acknowledge support from the European Research Council (ERC) under the European Union's Horizon 2020 
research and innovation program (ERC-StG-Neupert-757867PARATOP) and from the NCCR MARVEL funded of the Swiss National Science Foundation. S.S.T. is additionally sup- ported by Grant No. PP00P2_176877 from the Swiss National Science Foundation. X.L. was supported by the China Scholarship Council (CSC).
[1] C. L. Kane and E. J. Mele, Phys. Rev. Lett. 95, 146802 (2005).

[2] X.-L. Qi, T. L. Hughes, and S.-C. Zhang, Phys. Rev. B 78, 195424 (2008).

[3] A. Kitaev, in Advances in Theoretical Physics: Landau Memorial Conference, AIP Conf. Proc. No. 1134 (AIP, Melville, NY, 2009), p. 22.

[4] S. Ryu, A. P. Schnyder, A. Furusaki, and A. W. W. Ludwig, New J. Phys. 12, 065010 (2010).

[5] L. Fu, Phys. Rev. Lett. 106, 106802 (2011).

[6] Z. Wang, A. Alexandradinata, R. J. Cava, and B. A. Bernevig, Nature (London) 532, 189 (2016).

[7] J. Kruthoff, J. de Boer, J. van Wezel, C. L. Kane, and R.-J. Slager, Phys. Rev. X 7, 041069 (2017).

[8] H. C. Po, A. Vishwanath, and H. Watanabe, Nat. Commun. 8, 50 (2017).

[9] B. Bradlyn, L. Elcoro, J. Cano, M. G. Vergniory, Z. Wang, C. Felser, M. I. Aroyo, and B. A. Bernevig, Nature (London) 547, 298 (2017).

[10] F. Schindler, Z. Wang, M. G. Vergniory, A. M. Cook, A. Murani, S. Sengupta, A. Y. Kasumov, R. Deblock, S. Jeon, I. Drozdov, H. Bouchiat, S. Guéron, A. Yazdani, B. A. Bernevig, and T. Neupert, Nat. Phys. 14, 918 (2018).

[11] M. G. Vergniory, L. Elcoro, C. Felser, N. Regnault, B. A. Bernevig, and Z. Wang, Nature (London) 566, 480 (2019).

[12] A. Bouhon, A. M. Black-Schaffer, and R.-J. Slager, Phys. Rev. B 100, 195135 (2019).

[13] M. C. Rechtsman, J. M. Zeuner, Y. Plotnik, Y. Lumer, D. Podolsky, F. Dreisow, S. Nolte, M. Segev, and A. Szameit, Nature (London) 496, 196 (2013).

[14] C. L. Kane and T. C. Lubensky, Nat. Phys. 10, 39 (2014).

[15] R. Süsstrunk and S. D. Huber, Science 349, 47 (2015).

[16] M. Serra-Garcia, V. Peri, R. Süsstrunk, O. R. Bilal, T. Larsen, L. G. Villanueva, and S. D. Huber, Nature (London) 555, 342 (2018).

[17] V. Peri, Z.-D. Song, M. Serra-Garcia, P. Engeler, R. Queiroz, X. Huang, W. Deng, Z. Liu, B. A. Bernevig, and S. D. Huber, Science 367, 797 (2020).

[18] A. A. Burkov, M. D. Hook, and L. Balents, Phys. Rev. B 84, 235126 (2011).

[19] C. Fang, H. Weng, X. Dai, and Z. Fang, Chin. Phys. B 25, 117106 (2016).

[20] R. Yu, Z. Fang, X. Dai, and H. Weng, Front. Phys. 12, 127202 (2017).

[21] Y. Kim, B. J. Wieder, C. L. Kane, and A. M. Rappe, Phys. Rev. Lett. 115, 036806 (2015).

[22] J. Zak, Phys. Rev. Lett. 62, 2747 (1989).

[23] M. V. Berry, Proc. R. Soc. London, Ser. A 392, 45 (1984).

[24] T. Bzdušek, Q.-S. Wu, A. Rüegg, M. Sigrist, and A. A. Soluyanov, Nature (London) 538, 75 (2016).

[25] Z. Yan, R. Bi, H. Shen, L. Lu, S.-C. Zhang, and Z. Wang, Phys. Rev. B 96, 041103(R) (2017).

[26] R. Bi, Z. Yan, L. Lu, and Z. Wang, Phys. Rev. B 96, 201305(R) (2017).
[27] M. Ezawa, Phys. Rev. B 96, 041202(R) (2017).

[28] Z. Zhu, G. W. Winkler, Q. S. Wu, J. Li, and A. A. Soluyanov, Phys. Rev. X 6, 031003 (2016).

[29] J. Wang, X. Sui, W. Shi, J. Pan, S. Zhang, F. Liu, S.-H. Wei, Q. Yan, and B. Huang, Phys. Rev. Lett. 119, 256402 (2017).

[30] J.-Z. Ma, J.-B. He, Y.-F. Xu, B. Q. Lv, D. Chen, W.-L. Zhu, S. Zhang, L.-Y. Kong, X. Gao, L.-Y. Rong, Y.-B. Huang, P. Richard, C.-Y. Xi, E. S. Choi, Y. Shao, Y.-L. Wang, H.-J. Gao, X. D. Dai, C. Fang, H.-M. Weng, G.-F. Chen, T. Qian, and H. Ding, Nat. Phys. 14, 349 (2018).

[31] J. Kim, H.-S. Kim, and D. Vanderbilt, Phys. Rev. B 98, 155122 (2018).

[32] T. T. Heikkilä and G. E. Volovik, New J. Phys. 17, 093019 (2015).

[33] A. Das and S. Pujari, Phys. Rev. B 102, 235148 (2020).

[34] X. Wan, A. M. Turner, A. Vishwanath, and S. Y. Savrasov, Phys. Rev. B 83, 205101 (2011).

[35] S.-Y. Xu et al., Science 349, 613 (2015).

[36] B. Q. Lv, H. M. Weng, B. B. Fu, X. P. Wang, H. Miao, J. Ma, P. Richard, X. C. Huang, L. X. Zhao, G. F. Chen, Z. Fang, X. Dai, T. Qian, and H. Ding, Phys. Rev. X 5, 031013 (2015).

[37] A. A. Soluyanov, D. Gresch, Z. Wang, Q.-S. Wu, M. Troyer, X. Dai, and B. A. Bernevig, Nature (London) 527, 495 (2015).

[38] S. M. Young, S. Zaheer, J. C. Y. Teo, C. L. Kane, E. J. Mele, and A. M. Rappe, Phys. Rev. Lett. 108, 140405 (2012).

[39] B.-J. Yang and N. Nagaosa, Nat. Commun. 5, 4898 (2014).

[40] Z. K. Liu, B. Zhou, Y. Zhang, Z. J. Wang, H. M. Weng, D. Prabhakaran, S.-K. Mo, Z. X. Shen, Z. Fang, X. Dai, Z. Hussain, and Y. Chen, Science 343, 864 (2014).

[41] D. T. Son and B. Z. Spivak, Phys. Rev. B 88, 104412 (2013).

[42] X. Huang, L. Zhao, Y. Long, P. Wang, D. Chen, Z. Yang, H. Liang, M. Xue, H. Weng, Z. Fang, X. Dai, and G. Chen, Phys. Rev. X 5, 031023 (2015).

[43] X. Zhang, Z.-M. Yu, X.-L. Sheng, H. Y. Yang, and S. A. Yang, Phys. Rev. B 95, 235116 (2017).

[44] T.-T. Zhang, Z.-M. Yu, W. G. Guo, D. Shi, G. Zhang, and Y. Yao, J. Phys. Chem. Lett. 8, 5792 (2017).

[45] Y. Xie, J. Cai, J. Kim, P.-Y. Chang, and Y. Chen, Phys. Rev. B 99, 165147 (2019).

[46] L. Jin, X. Zhang, X. Dai, H. Liu, G. Chen, and G. Liu, J. Mater. Chem. C 7, 1316 (2019).

[47] L. Jin, X. Zhang, T. He, W. Meng, X. Dai, and G. Liu, Phys. Chem. Chem. Phys. 22, 5847 (2020).

[48] G. P. Mikitik and Y. V. Sharlai, Phys. Rev. B 73, 235112 (2006).

[49] C. Fang, Y. Chen, H.-Y. Kee, and L. Fu, Phys. Rev. B 92, 081201(R) (2015).

[50] T. Bzdušek and M. Sigrist, Phys. Rev. B 96, 155105 (2017).

[51] J. Ahn, S. Park, D. Kim, Y. Kim, and B.-J. Yang, Chin. Phys. B 28, 117101 (2019).

[52] E. Lee, R. Kim, J. Ahn, and B.-J. Yang, npj Quantum Mater. 5, 1 (2020).

[53] J. Ahn, D. Kim, Y. Kim, and B.-J. Yang, Phys. Rev. Lett. 121, 106403 (2018). 
[54] A. Tiwari and T. Bzdušek, Phys. Rev. B 101, 195130 (2020).

[55] Q. Wu, A. A. Soluyanov, and T. Bzdušek, Science 365, 1273 (2019).

[56] J. Ahn, S. Park, and B.-J. Yang, Phys. Rev. X 9, 021013 (2019).

[57] A. Bouhon, Q. Wu, R.-J. Slager, H. Weng, O. V. Yazyev, and T. Bzdušek, Nat. Phys. 16, 1137 (2020).

[58] E. Yang, B. Yang, O. You, H.-C. Chan, P. Mao, Q. Guo, S. Ma, L. Xia, D. Fan, Y. Xiang, and S. Zhang, Phys. Rev. Lett. 125, 033901 (2020).

[59] J. Kang and O. Vafek, Phys. Rev. B 102, 035161 (2020).

[60] C. Zhong, Y. Chen, Z.-M. Yu, Y. Xie, H. Wang, S. A. Yang, and S. Zhang, Nat. Commun. 8, 15641 (2017).

[61] C. J. Bradley and A. P. Cracknell, The Mathematical Theory of Symmetry in Solids: Representation Theory for Point Groups and Space Groups (Clarendon, Oxford, 1972).

[62] M. I. Aroyo, J. M. Perez-Mato, C. Capillas, E. Kroumova, S. Ivantchev, G. Madariaga, A. Kirov, and H. Wondratschek, Z. Kristallogr. Cryst. Mater. 221, 15 (2006).

[63] M. I. Aroyo, A. Kirov, C. Capillas, J. M. Perez-Mato, and H. Wondratschek, Acta Crystallogr., Sect. A 62, 115 (2006).

[64] L. Elcoro, B. Bradlyn, Z. Wang, M. G. Vergniory, J. Cano, C. Felser, B. Andrei Bernevig, D. Orobengoa, G. De La Flor, and M. I. Aroyo, J. Appl. Crystallogr. 50, 1457 (2017).

[65] D. Gresch, Ph.D. thesis, ETH Zurich, 2017.

[66] P. M. Lenggenhager, X. Liu, S. S. Tsirkin, and T. Neupert, and T. Bzdušek, Data and Code for: Multi-Band Nodal Links in Triple-Point Materials (ETH, Zurich, 2020), doi:10.3929/ethzb-000423924.

[67] Z. Yang, C.-K. Chiu, C. Fang, and J. Hu, Phys. Rev. Lett. 124, 186402 (2020).
[68] P. M. Lenggenhager, X. Liu, S. S. Tsirkin, T. Neupert, and T. Bzdušek (unpublished).

[69] See Supplemental Material at http://link.aps.org/supplemental/ 10.1103/PhysRevB.103.L121101, which includes Refs. [78-85], for additional information about the construction of the tight-binding model of $\mathrm{Li}_{2} \mathrm{NaN}$ and the study of its properties, in particular the surface states.

[70] R. Kubo, J. Phys. Soc. Jpn. 12, 570 (1957).

[71] D. A. Greenwood, Proc. Phys. Soc. 71, 585 (1958).

[72] S. S. Tsirkin, NPJ Comput. Mater. 7, 33 (2021).

[73] S. Barati and S. H. Abedinpour, Phys. Rev. B 96, 155150 (2017).

[74] X.-Q. Sun, S.-C. Zhang, and T. Bzdušek, Phys. Rev. Lett. 121, 106402 (2018).

[75] M. Lin and T. L. Hughes, Phys. Rev. B 98, 241103(R) (2018).

[76] Z. Wang, B. J. Wieder, J. Li, B. Yan, and B. A. Bernevig, Phys. Rev. Lett. 123, 186401 (2019).

[77] B. J. Wieder, Z. Wang, J. Cano, X. Dai, L. M. Schoop, B. Bradlyn, and B. A. Bernevig, Nat. Commun. 11, 627 (2020).

[78] G. Kresse and J. Furthmüller, Phys. Rev. B 54, 11169 (1996).

[79] G. Kresse and D. Joubert, Phys. Rev. B 59, 1758 (1999).

[80] J. P. Perdew, K. Burke, and M. Ernzerhof, Phys. Rev. Lett. 77, 3865 (1996).

[81] J. Gao, Q. Wu, C. Persson, and Z. Wang, Comput. Phys. Comm. 261, 107760 (2021).

[82] Q. Wu, S. Zhang, H.-F. Song, M. Troyer, and A. A. Soluyanov, Comput. Phys. Commun. 224, 405 (2018).

[83] N. Marzari and D. Vanderbilt, Phys. Rev. B 56, 12847 (1997).

[84] G. Pizzi et al., J. Phys.: Condens. Matter 32, 165902 (2020).

[85] D. Varjas, T. Ö. Rosdahl, and A. R. Akhmerov, New J. Phys. 20, 093026 (2018). 